\title{
¿Jugamos al Súper Mario Bros? Descripción de una experiencia gamificada en la formación del profesorado de Educación Física \\ Will we play Super Mario Bros? Description of a gamified experience in the training of Physical Education teachers \\ Gonzalo Flores Aguilar \\ Universidad de Barcelona (España), Universidad de Vic (España)
}

\begin{abstract}
Resumen. De cara a aumentar e impulsar la presencia de las metodologías activas en la enseñanza de la Educación Física, en este artículo se describen las características básicas de una experiencia gamificada denominada «Súper Mario Bros», que ha sido desarrollada y aplicada durante el curso 2017/2018 en una de las asignaturas del Grado de Ciencias de la Actividad Física y del Deporte (CAFE). Además de detallar los «ingredientes clave» de los que se compone la gamificación, y la manera en la que el docente los incorpora en su experiencia, aquí se presentan un conjunto de reflexiones sobre diversos aspectos a tener en cuenta en cada uno de dichos ingredientes. En definitiva, con este trabajo se quiere difundir la manera en la que se puede gamificar una asignatura, a partir de una experiencia real y exitosa, que puede servir como modelo inspirador para el profesorado interesado.
\end{abstract}

Palabras clave: aprendizaje cooperativo, educación física, formación del profesorado, gamificación, hibridación.

Abstract. In order to increase and promote the presence of active methodologies in the teaching of Physical Education, this article describes the basic characteristics of a gamified experienced called «Super Mario Bros», which has been developed and implemented during the course 2017/2018 in one of the subjects of the Degree program in Sciences of Physical Activity and Sports (CAFE). In addition to detailing the «key ingredients» which gamification is composed of, and the way teachers incorporate them into their projects, here are a set of reflections on various aspects to consider in each of those ingredients. In short, this work aims to disseminate the way in which a subject can be gamified, based on a real and successful experience, which can serve as an inspiring model for interested teachers.

Key words: cooperative learning, physical education, gamification, hybridization, teacher training.

\section{Introducción}

La incorporación de la gamificación en el mundo educativo está en auge ya que se considera una metodología innovadora y atractiva para el alumnado del siglo XXI (Llopis \& Balaguer, 2017). También conocida como ludificación, ambos conceptos provienen del anglicismo Gamification (Kapp, Blair \& Mesch, 2014). Aunque este término surge en el año 2008, no es hasta el 2010 cuando se empieza a generalizar en la educación y en otros ámbitos, como el empresarial, la salud, el marketing, etc. (Melchor, 2012).

A pesar que actualmente todavía no existe un conceso definitorio (Torres, Romero, Pérez \& Björk, 2018), y a partir de la revisión bibliográfica llevada a cabo, la gamificación en educación podría entenderse como una metodología donde se utiliza una historia o narrativa imaginaria, como hilo conductor, con el fin de consolidar las competencias u objetivos de aprendizaje a partir de la introducción de las mecánicas de los juegos o videojuegos (retos, misiones, recompensas, etc.); por cierto, sin necesidad de utilizar un producto comercial (Gómez, Molina \& Devís, 2018). Para ello, la gamificación incide directamente en los aspectos emocionales, motivacionales y comportamentales del alumnado (Llopis \& Balaguer, 2017), al convertir el proceso de enseñanza-aprendizaje en un elemento nuevo, atractivo y, en última instancia, mucho más efectivo (Torres et al., 2018).

En palabras de Ayén (2017, p.8):

Con la gamificación se pretende generar en el alumno las mismas emociones y sentimientos que siente con los juegos a los que se aficiona, con el fin de engancharlo al proceso de aprendizaje. Se trata de intentar reproducir en

Fecha recepción: 04-10-18. Fecha de aceptación: 25-01-19 Gonzalo Flores Aguilar gonzalo.flores@ub.edu clase la magia, los sueños, la sensación de superación, la desconexión mediante realidades de ficción distintas a las cotidianas. Una gamificación exitosa es aquella en la que el alumno se mueve por motivación intrínseca, es decir, porque disfruta con lo que está haciendo, a diferencia de la motivación extrínseca, basada en la realización de actividades que no se consideran agradables, ya sea por la búsqueda del premio o por el temor al castigo. Según los trabajos publicados, la gamificación suele generar un alto grado de compromiso e implicación hacia la asignatura en el alumnado (Cortizo, Carrero, Monsalve, Velasco, Díaz \& Pérez, 2011; Hanus y Fox, 2015; entre otros), ya que la motivación intrínseca y extrínseca puede aumentar, aunque existan algunos detractores (Carrasco, Matamoros \& Flores, 2019; Chan, Fui-Hoon, Liu \& Lu, 2018), al igual que los componentes afectivos y emocionales positivos (excitación, sorpresa, curiosidad, felicidad, alegría, etc.) (Llopis \& Balaguer, 2017). A su vez, Cortizo et al. (2011) afirman que la gamificación mejora los niveles de colaboración y la resolución de problemas, puesto que se disminuye el miedo a equivocarse.

En este sentido, la revisión bibliográfica revela la existencia de un conjunto de estudios o experiencias nacionales donde se difunden los resultados que se derivan de la aplicación de la gamificación en educación primaria y secundaria (Carrasco et al., 2019; Fernández-Gavira, Prieto, Alcaraz, Sánchez-Oliver \& Grimaldi, 2018; Martín \& Ruiz, 2016; Martínez-Franco, 2017; Monguillot, Arévalo, Mon, Batet \& Catasús, 2015; Pisabarro \& Vivaracho, 2018; Quintero, Jiménez \& Area, 2018) y en educación superior (Corchuelo, 2018; Chan et al., 2018; Ferrerira \& Lacerda, 2018; Navarro, Martínez \& Pérez-López, 2017; Pérez-López, 2009; Pérez-López \& Rivera-García, 2017; Pérez-López, Rivera-García \& Delgado, 2017; Pérez-López, Rivera \& Trigueros, 2017). 
Melchor (2012) subraya que, para que la gamificación tenga éxito, los proyectos gamificados deben ser atractivos y deben despertar el interés del alumnado; deben ofrecer recompensas que permitan implicarlos al proceso; y deben ser suficientemente flexibles para utilizarse tanto de forma individual como colectiva dentro del aula (Íbid.).

Para ello, y en cuanto a los procesos de creación de una experiencia gramificada se refiere, existen diversos elementos a tener en cuenta. Por ejemplo, Werbach \& Hunter (2012) establecen tres niveles, en forma de pirámide, donde se incluyen un conjunto de dinámicas (aspectos narrativos, emociones, límites, progresión o interacciones); mecánicas (recompensas, retroalimentaciones, retos, competición y cooperación); y componentes (avatares, insignias, puntos, combates, rankings clasificatorios, niveles, equipos). Ripoll (2017) considera, de un modo más simplificado, que la gamificación debe componerse de los siguientes ingredientes clave: mundo-narrativa, misión, niveles, recompensas, avatares, equipos, puntos de experiencia, bienes, clasificación, eventos especiales, medallas-trofeos y área social.

Según un reciente estudio de Kocakoyun \& Ozdamli (2018), actualmente España ocupa el segundo lugar, tras EEUU, dentro del conjunto de países con más publicaciones activas (Web of Science) sobre la temática. Sin embargo, la mayoría de estos trabajos se centran en ámbitos médicos, tecnológicos o empresariales, y no tanto en el ámbito educativo o en la formación del profesorado. Por este motivo, avanzar en la investigación y en la aplicación y posterior divulgación de experiencias gamificadas en educación, y concretamente en Educación Física (EF), independientemente de su nivel educativo (primaria, secundaria, bachillerato, ciclos formativos y formación del profesorado), se considera un reto y una necesidad de primer orden.

\section{Descripción de la experiencia «Súper Mario Bros»}

Inicialmente esta experiencia nació con el propósito inicial de incrementar el grado motivación del alumnado participante, además de su rendimiento académico general. De igual modo, y a partir de la vivenciación del mismo, con esta experiencia se busca despertar el interés del futuro profesorado de EF hacia la gamificación.

Las personas participantes fueron los 76 estudiantes matriculados en la asignatura de «Didáctica de la Educación Física II» de tercero del Grado de Ciencias de la Actividad Física y del Deporte (CAFE) que se impartió en la Universidad de Vic-Universidad Central de Cataluña (UVIC-UCC), durante el curso 2017-2018; además del docente que imparte dicha materia.

Cabe destacar que esta experiencia incorpora la gamificacion a lo largo de toda la asignatura (los seis créditos ECTS), aunque esto no es siempre obligatorio. Pueden gamificarse bloques de contenidos, unidades didácticas, semestres, etc. Según Ripoll (2017), es mejor comenzar de menos a más.

Ante este panorama, y en el marco de un proyecto global donde se realiza una investigación-acción que incluye el estudio en profundidad de la visión del alumnado y del profesorado sobre esta experiencia y sus efectos, en este trabajo se describen las particularidades más intimas de «Súper
Mario Bros» utilizando como hilo conductor los ingredientes clave de la gamificación utilizados (Ripoll, 2017), a la vez que se hace referencia a los elementos básicos del Aprendizaje Cooperativo (Johnson, Johnson y Holubec, 2013), puesto que en esta experiencia ambas metodologías han sido hibridadas.

De igual modo, al final de la explicación de cada uno de los ingredientes se ofrecen algunas reflexiones personales del docente encargado, en función de su propia experiencia, que pueden ayudar a las personas lectoras a ampliar la información sobre el tema.

\section{Ingrediente 1: Mundo-Narrativa}

El espacio imaginario donde transcurre esta experiencia, y por tanto, el desarrollo de la asignatura, es el mítico videojuego «Súper Mario Bros».

En primer lugar, la elección de esta temática se debe a la inspiración que suscitó el trabajo de Martínez-Franco (2017). De igual modo, los motivos finales que justifican la selección de este mundo son los siguientes: a) conocimiento y vivencias positivas por parte del alumnado universitario; b) objetivo final del juego claro; c) personajes y niveles ya existentes; d) dinámica de recompensas y premios transferibles.

Con tal de conseguir un gran impacto inicial en el alumnado, además de crear un logo específico, el aula donde se desarrollan las sesiones teóricas fue decorada con imágenes del juego. Caba decir también que el docente asistió a la mayoría de sesiones con una camiseta temática (figura 1).

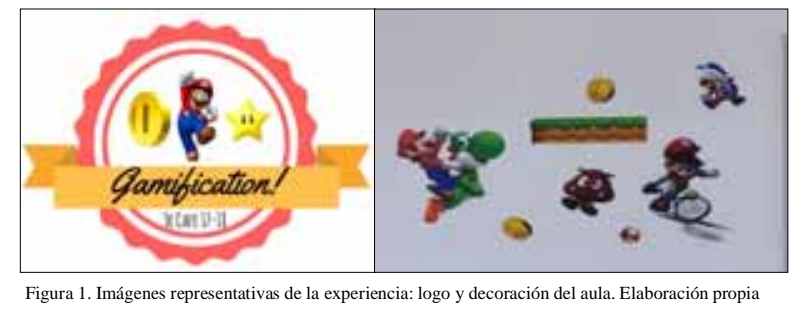

Reflexiones del docente sobre el ingrediente 1

Después de llevar a cabo otra experiencia gamificada el pasado curso académico, a modo de prueba piloto (Flores \& Prat, 2018), cabe subrayar que la elección de un buen mundo o narrativa es un aspecto clave para su adecuado desarrollo (Melchor, 2012). En este sentido, es importante ser creativo a la hora de invertarse mundos propios, pero esto no es indispensable. Si se sufre de periodos de poca creatividad, siempre puedes inspirarte en otros mundos ya publicados. De igual modo, es importante conocer los gustos del alumnado, las modas del momento, y profundizar en ellas. En el caso de esta experiencia, el docente estuvo las semanas previas jugando al videojuego de Mario Bros con la intención de familiarizarse con el mismo, además de haberse leído una enciclopedia temática ya publicada (VV.AA, 2017).

En último lugar, la puesta en escena de la experiencia gamificada también adquiere un especial interés. Es importante impactar al alumnado, y también generarle curiosidad y ganas de empezar. En este caso, aquí se recurrió a la música temática del videojuego (se utilizó todos los días antes de empezar la sesión) además de una pequeña dramatización del docente que causó mucho agrado. El objetivo era simular que el villano del videojuego (Bowser) había secuestrado a 
la princesa (ver ingrediente 2) y que los miembros de la clase deberían salvarla en un juego por equipos.

\section{Ingrediente 2: Misión-Retos}

$\mathrm{Al}$ igual que en el videojuego, el objetivo final de esta experiencia consiste en intentar salvar a la princesa Peach, la cual se encuentra retenida en el castillo final por el maléfico Bowser (rol que ejerce el docente). Para ello será necesario superar un conjunto de retos según cada nivel.

Estos retos incluyen actividades individuales y actividades grupales, tales como la lectura de artículos, la elaboración de las diferentes partes de una unidad didáctica, la elaboración de instrumentos de evaluación, etc.; además de la evaluación de la actitud del alumnado.

\section{Reflexiones del docente sobre el ingrediente 2}

No hay que olvidar que el verdadero objetivo de la gamificación en educación es que el alumnado adquiera las competencias y los resultados de aprendizaje de la asignatura. Esta es la verdadera misión final. El mundo de Mario Bros es el atrezo o la burbuja en la que se enmarca la asignatura.

Los retos iniciales deberían ser accesibles y progresivos en dificultad. Es importante no realizar demasiados retos puesto que el alumnado puede llegar a agobiarse. Mejor realizar pocos retos y que sean fácilmente evaluables. En este caso, los feedbacks de los mismos adquieren un especial interés, por ello la evaluación formativa debería ser el tipo de evaluación en la que se enmarquen las materias gamificadas.

La planificación previa de todos los retos, para evitar la improvisación, es otro aspecto clave. El alumnado, antes de empezar cada nivel, debe saber cómo puede supéralo, es decir, debe saber cuantos retos debe realizar y en qué tiempo.

Finalmente, resaltar también la posibilidad de modificar en cada proyecto todas aquellas actitudes sexistas o violentas que el propio Mundo (en este caso el videojuego del Mario Bros), pudiese tener per se. En vez de salvar a la princesa a través de una batalla, podría hablarse mejor de llegar al mundo final y conseguir entrar en el castillo, por ejemplo.

\section{Ingrediente 3: Niveles}

En base al juego oficial, en esta experiencia se han generado tres niveles o fases antes de la «batalla final». Cada nivel corresponde a un tema de la asignatura, y todos y cada uno de los retos a superar son actividades propias de la misma (tabla 1). Para más información sobre la materia puede accederse a su plan de estudios: https://www.uvic.cat/ assignatura/1176

\section{Reflexiones del docente sobre el ingrediente 3}

Después de esta experiencia, se recomienda crear tantos niveles como temas o unidades didácticas pueda tener la asignatura, el trimestre o semestre. Esto facilita mucho la elaboración de los retos, puesto que estos pueden basarse en las actividades más adecuadas con las que los estudiantes podrán adquirir las competencias correspondientes.

A su vez, visualizar los niveles de modo gráfico o digital, en este caso en forma de diapositivas colgadas en la pared del aula, ayuda a ubicar al alumnado, de la misma manera que

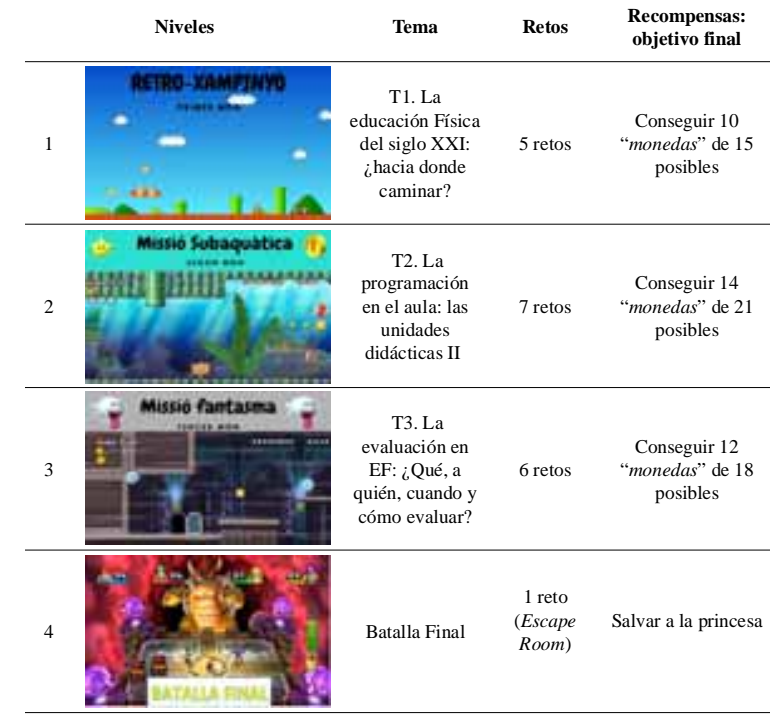

lo motiva. Para esta creación audiovisual se utilizó el programa Canva, y se recurrió a los fotogramas del juego original que están accesibles en la plataforma Youtube.

\section{Ingredientes 4 y 5: Avatares-jugadores y equipos}

Siguiendo las características del Aprendizaje Cooperativo, se crearon grupos heterogéneos de cuatro y/o cinco miembros. Cada grupo correspondía a un personaje del juego (figura 2), el cual debían personalizar mediante una ficha, la cual les serviría para colocarse en el tablero (colgado en la pared).

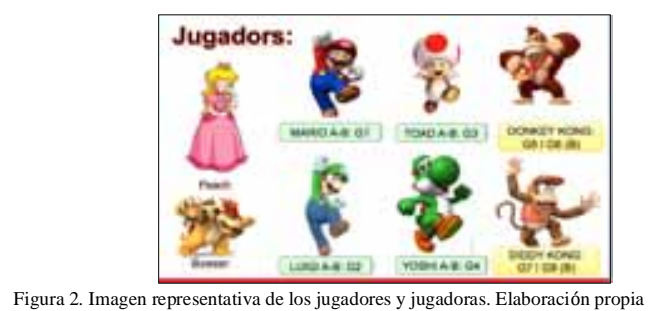

Cada grupo firmó un «compromiso de trabajo en grupo», donde se comprometían a trabajan colectivamente, a ayudarse, a motivar a quién lo necesite, e informar al docente en caso de omnipresencia (interdependencia positiva-responsabilidad individual) (Johnson, Johnson y Holubec, 2013). También se comprometían ua efectuar una autoevaluación de cada etapa, además del reparto de la nota final en función de trabajo real realizado por cada uno de ellos (procesamiento grupal-interacción promotora) (Ibíd.).

Además, el docente también recurre a la aplicación Classdojo como plataforma de comunicación para el aula que permite conectar a docentes, alumnado y familiares para compartir mensajes, fotos y vídeos, de manera instantánea. A través de los avatares que esta misma aplicación genera y otoga automáticamente a cada estudiante, en forma de monstruo, el docente puede introducir y puntuar, positiva o negativamente, un conjunto de habilidades y/o comportamientos deseables. Gracias a ello, el docente puede evaluar y calificar mejor, por ejemplo, la actitud del alumnado hacia la asignatura. 
Reflexiones del docente sobre los ingredientes 4 y 5

La creación de grupos heterogéneos ha sido un problema en todo momento. A pesar de todas las estrategias empleadas, finalmente existieron grandes resistencias. De hecho, el docente se vió obligado a deshacer tres equipos por el incumplimiento del «compromiso de trabajo en grupo» firmado a principio de curso. Aunque esto ralentice las correcciones y los feedbacks, de esta manera se intenta asegurar una participación un poco más equitativa. Sobre esto, y a pesar de las dificultades, se recuerda la importancia de seguir apostando por la creación de pequeños grupos heterogéneos.

En último lugar, resaltar la utilidad del programa Classdojo como elemento clave para el seguimiento de la materia así como para la comunicación instantánea con el alumnado.

\section{Ingrediente 6: Recompensas}

Son varias las recompensas que podían aquí obtenerse. Además de las típicas «monedas» que se obtienen con cada reto (figura 3), el grupo que era capaz de pasar de nivel podía obtener una «llave secreta» que le permitía entrar en el siguiente nivel, y que le sería de gran ayuda para el último nivel (la batalla final) (en el caso de que ese grupo pudiera llegar).

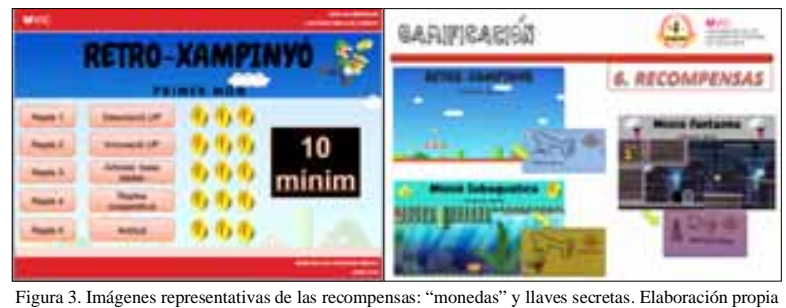

\section{Ingredientes 7 y 8: Puntos de experiencia y bienes}

El procedimiento de otorgación y revisión de las «monedas» ha sido el siguiente. Al principio de cada tema el docente explicó todos los retos y todas las «monedas» que se podían y se necesitaban conseguir para pasar de nivel. Una vez acordados lo términos o plazos de entrega, y en el plazo máximo de una semana tras la finalización de cada nivel, el docente se comunicaba con cada uno de los equipos para ofrecerles el feedback de sus tareas y para detallar el conjunto de «monedas» obtenidas. En función de cada reto, el criterio seguido es el siguiente: 0 «monedas»= mal trabajo; 1 «moneda»= trabajo débil con muchas mejoras; 2 «monedas»= trabajo aceptable pero con algunas mejoras; 3 «mone$d a s »=$ trabajo muy bueno, quizás alguna leve mejora).

\section{Reflexiones del docente sobre el ingrediente 6}

Independientemente de las «monedas» obtenidas, un aspecto clave fue la recuperación de las mismas. Para mantener la motivación y el ritmo del juego, y evitar así que los equipos se descolgasen, tras la finalización de los niveles el docente otorgaba diversos plazos, normalmente de una semana, para que los equipos rezagados sí superaran el nivel. Esto es un claro ejemplo de la evaluación formativa empleada.

También hay que destacar la función de las «llaves secretas» (tarjetas de colores impresas). Este material, del que el alumnado debía responsabilizarse, era objeto de deseo y cuidado por parte de los equipos; una idea también subrayada por Melchor (2012) y Monguillot et al. (2015).

Ingredientes 7 y 8: Puntos de experiencia y bienes

Los jugadores, a título individual, tenían la posibilidad de realizar «actividades extra» (para saber más) que aparecían sin previo aviso en el campus virtual (lectura de artículos, escucha de podcasts, visualización de vídeos, etc.). En cada nivel había un total de tres «actividades extra». Por cada tres actividades realizadas correctamente se entregaban dos «estrellas». Cuando se recopilaban tres «estrellas» se obtenía una carta sorpresa que incluía un premio (puntuación extra, elección de preguntas para el examen, etc.) (figura 4).

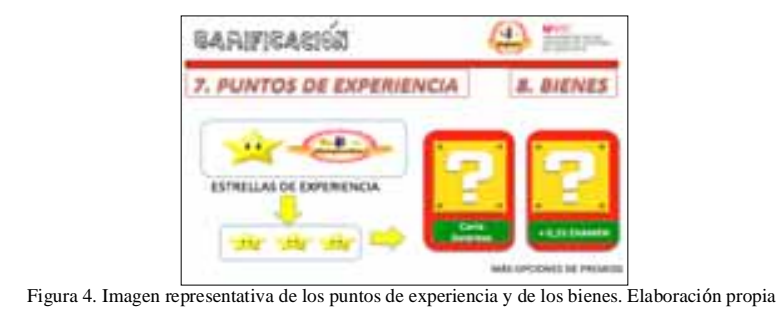

Reflexiones del docente sobre los ingredientes 7 y 8

Llama la atención el elevado grado de motivación que presentaba una gran parte del grupo por realizar estas «actividades extras». Sorprendentemente, casi un $40 \%$ de las personas participantes cumplieron con estas tareas optativas, sobre todo por el gran deseo que les generó poder conseguir las «estrellas» y las «cartas sorpresas». Como incican Pérez-López y Rivera García (2017), existe una relación directa entre la existencia de emociones positivas y la implicación por el trabajo.

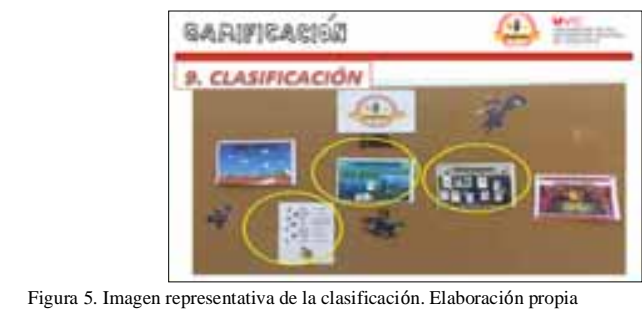

\section{Ingrediente 9: Clasificación}

A pasar del vandalismo vivido en el aula, visualizar públicamente la posición de los equipos durante el transcurso del juego fue importante para el mantenimiento del interés y la motivación de los equipos (figura 5).

\section{Reflexiones del docente sobre el ingrediente 9}

Para la clasificación de los resultados se utilizó el tablón de corcho del aula, a pesar de las dificultades existentes (el personal de mantenimiento o el alumnado de otras asignaturas realizaron cambios sin permido del docente en todas las paredes del aula). Para evitar esto, en futuras ocasiones sería interesante utilizar el programa Genially, ya que es posible generar un tablero online donde reflejar la posición de cada equipo.

Ingrediente 10: Eventos especiales

En un total de cinco ocasiones el docente recurrió a los 
«eventos especiales» o sorpresas para romper con la rutina de clase (figura 6). Este tipo de actividades ayudan a mantener la concentración y a mejorar la implicación y la motivación hacia la experiencia. Cabe decir que con la mayoría de estos eventos se podían obtener «premios» individuales o grupales.

El evento número uno consistió en ir a buscar unas piezas gigantes en forma de «estrella» que estaban escondidas por el campus. El alumnado supo de dicha actividad antes de empezar la sesión del aula ordinaria. El grupo tuvo 5 minutos para intentar encontrarlas.

El evento número dos consistió en realizar diferentes cuestionarios sorpresas utilizando la aplicación Kahoot. Estos cuestionarios contenían preguntas relativas al contenido de la asignatura. Las tres primeras personas de la clasificación obtuvieron «monedas» extras para su grupo.

Lejos de obtener algún premio, con el evento tres los jugadores y jugadoras participaban en la sesión teórica de la asignatura en un baile grupal, a modo de pausa activa, a partir de la utilización de los videos musicales de Just Dance colgados en Youtube.

El evento con más éxito fue el número cuatro. Con las posibles preguntas que los equipos habían creado en la aplicación Kahoot para un hipotético examen, el día del evento se realizó una batalla, a modo de desafíos por equipos. Los equipos se retaban entre sí para ver cual de ellos acertaba más preguntas del contrincante, y viceversa. Los equipos que ganaban y perdían subían o bajaban de nivel, respectivamente, utilizando la pirámide de niveles dibujada en la pizarra. Al finalizar el juego, los equipos presentes en la cima conseguían «estrellas» extra.

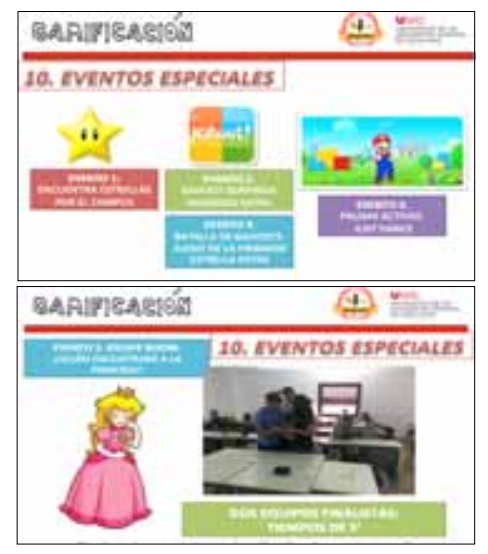

Figura 6. Imágenes representativas de los eventos especiales. Elaboración propia

El último evento consistió en la participación de un Escape Room. Este evento correspondía al último nivel del juego, y no todos los jugadores y jugadoras podían participar. Era importante acotar los grupos, en función de la puntuación global obtenida hasta el momento (número de monedas globales), para así conseguir un buen funcionamiento de la actividad (figura 7).

\section{Reflexiones del docente sobre el ingrediente 10}

Sin lugar a dudas este fue el ingrediente más divertido y gratificante. El alumnado no suele esperar este tipo de acciones, lo cual provoca grandes niveles de satisfacción y motivación (Melchor, 2012). De hecho, se ha podido comprobar

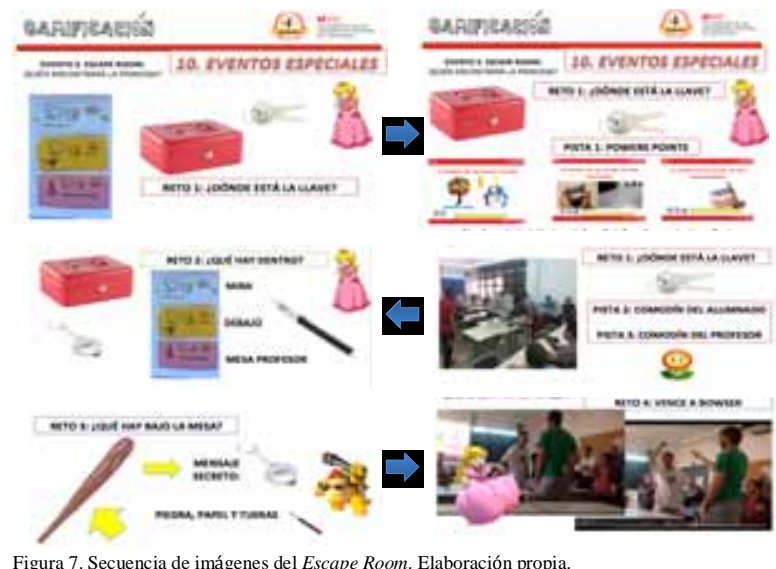

como estas pequeñas pausas ayudaban a mejorar el grado de atención posterior. El alumnado lo agradecía, y también lo reclamaba.

\section{Ingrediente 11: Área social}

Disponer de un espacio físico para facilitar las habilidades grupales y el contacto cara a cara es un ingrediente indispensable de la gamificación y el Aprendizaje Cooperativo (Johnson, Johnson y Holubec, 2013). En este caso, el punto de encuentro semanal era doble; por un lado el aula teórica (primera sesión semanal) y por el otro el gimnasio de la facultad (segunda sesión semanal).

\section{Reflexiones del docente sobre el ingrediente 11}

Bien es cierto que las sesiones del gimnasio parecían desconectadas de la experiencia gamificada, ya sea por la no decoración del mismo o por la no vinculación de retos específicos. Para evitarlo, el docente aludió en numerosas ocasiones a los retos, a las misiones, a los niveles, etc. con tal de ubicar al alumnado con la experiencia. En un futuro, sería interesante promover aquí también la consecución de premios extra durante estas sesiones prácticas.

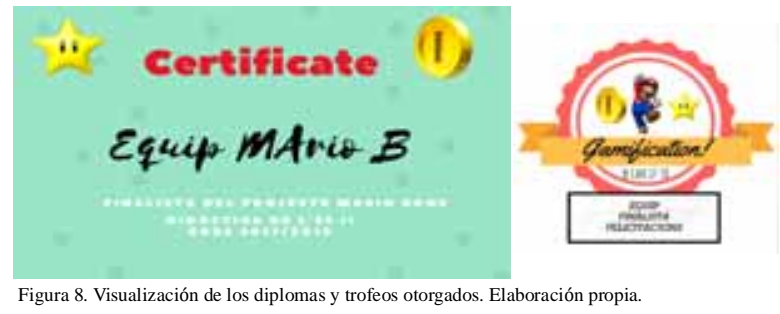

\section{Ingrediente 12: Medallas-premios}

En «Súper Mario Bros» se entregaron premios finales a los equipos que consiguieron salvar a la princesa, pero también a todos los participantes del mismo. El último día de clase se simuló la entrega de «premios» (trofeos de cartón) y se entregaron unos «diplomas acreditativos» (figura 8).

Reflexiones del docente sobre el ingrediente 12

Si se disponen de recursos económicos existen bastantes materiales que podrían ser utilizados como premios. Tampoco esto es indispensable. En este caso, todos los premios han sido caseros, y de bajo coste. Quizás, el hecho de entregar medallas de plástico, chapas, camisetas o trofeos temáticos, entre otros objetos, habría permitido «lucir» mucho más la experiencia. Aún así, el alumnado mostró mucha ilusión 
hacia los mismos llegando incluso a afirmar que era la primera vez que en la universidad, y en este caso en una asignatura, se les otorgó una distinción física.

\section{Conclusiones y líneas de futuro}

El desarrollo de una óptima experiencia gamificada requiere de una laboriosa preparación previa, sobre todo en cuanto a la presencia de la mayoría de los «ingredientes clave» ya descritos; además de en la creación del diseño de logos y materiales específicos vinculados con el «mundo» escogido. De igual modo, es muy aconsejable dominar o conocer las particularidades de dicho «mundo» (en este caso las características y dinámicas del Mario Bros), al igual que transmitir entusiamo y motivación al alumnado hacia todas las dinámicas.

Aún así, arriesgarse a desarrollar y aplicar una experiencia de este tipo en la formación del profesorado de EF se considera una experiencia muy positiva, que además se reedita curso tras curso, puesto que son muchas más las ventajas que los inconvenientes que se derivan de la misma. Para paliar las dificultades con las que se puede encontrar el docente, se recomienda basarse en otras experiencias gamificadas ya publicadas, sobre todo para el profesorado novel, además de colaborar con otros docentes afines. La familiarización con las dinámicas de los juegos (de mesa, de cartas, de rol, etc. y/o los videojuegos) también es un aspecto destacable. De igual modo, es mejor comenzar a gamificar de manera progresiva, es decir, empezar por algunas actividades o algunos temas, hasta llegar a gamificar, si se cree oportuno, incluso una unidad didáctica o trimestre.

Con todo ello, este trabajo se presenta como un ejemplo o modelo explicativo de los pasos a seguir para iniciarse en la gamificación que se espera que sirva de inspiración para el profesorado interesado.

\section{Referencias}

Ayén, F. (2017). ¿Qué es la gamificación y el ABJ? Íber Didáctica de las Ciencias Sociales, Geografía e Historia,86, 7-15

Carrasco, V.J., Matamoros, A. \& Flores, G. (2019). Analysis and comparison of the results obtained after the application of a gamified methodology and a traditional one in physical education in «bachillerato» (Spanish education for 16 to 18 years old students). ESHPA -Education, Sport, Health and Physical Activity, $3(1), 29-45$

Corchuelo, C.A. (2018). Gamificación en educación superior: experiencia innovadora para motivar estudiantes y dinamizar contenidos en el aula. EDUTEC. Revista Electrónica de Tecnología Educativa, 63, 29-41.

Cortizo, J. C., Carrero, F., Monsalve, B., Velasco, A., Díaz, L. I. \& Pérez, J. (2011). Gamificación y Docencia/ : Lo que la Universidad tiene que aprender de los Videojuegos. VIII Jornadas Internacionales de Innovación Universitaria Retos y oportunidades del desarrollo de los nuevos títulos en educación superior. Disponible en: http://abacus.universidadeuropea.es/bitstream/handle/ 11268/1750/46_Gamificacion.pdf?sequence $=2 \&$ is Allowed $=y$

Chan, E., Fui-Hoon, F., Liu, Q. \& Lu,Z. (2018). Effect of gamification on intrinsic motivation. En Nah FH., Xiao B. (eds) HCI in Business, Government, and Organizations. HCIBGO 2018. Lecture Notes in Computer Science, vol 10923. Springer, Cham

Fernández-Gavira, J., Prieto, E., Alcaraz, V., Sánchez-Oliver, A. \& Grimaldi, M. (2018). Aprendizajes significativos mediante la gamificación a partir del juego de rol: «Las Aldeas de la Historia». Espiral. Cuadernos del profesorado, 11(22), 69-78

Ferrerira, A. \& Lacerda, G. (2018). Gamificación como estrategia didáctica aplicación en la formación del profesor. Tendencias pedagógicas, 31, 113-125

Flores, G. \& Prat, M. (2018). «X-Vic: corre y vuela sobre los pirineos». Un proyecto gamificado y cooperativo en educación superior. En Fernández-Río y Méndez (coords). Actas del XI Congreso Internacional de Actividades Físicas Cooperativas, (697-709). Oviedo: ediciones de la universidad de Oviedo.

Gómez, F., Molina, P. \& Devís, J. (2018). Los videojuegos como materiales curriculares: una aproximación a su uso en Educación Física. Retos, 34, 305-310.

Hanus, M. D. \& Fox, J. (2015). Assessing the effects of gamification in the classroom: A longitudinal study on intrinsic motivation, social comparison, satisfaction, effort, and academic performance. Computers and Education, 80, 152-161.

Johnson, D. W., Johnson, R. T. \& Holubec, E. J. (2013). Cooperation in the Classroom (9th ed.). Edina, MN: Interaction Book Company.

Kapp, K.; Blair, L. \& Mesch, R (2014). The gamification of learning and instruction fieldbook. Ideas into practice. EEUU: Wiley.

Kocakoyun, S. \& Ozdamli, F. (2018). A Review of Research on Gamification Approach. En Rosalba Morese, Sara Palermo, Juri Nervo (Eds.), Socialization - A Multidimensional Perspective. DOI: 10.5772/intechopen.74131. Available from: https:// www.intechopen.com/books/socialization-a-multidimensionalperspective/a-review-of-research-on-gamification-approach-ineducation

Llopis, M.A. \& Balaguer, P. (2016). El uso del juego en educación. Gamificación. En Chiva, O. y Martí, M. (coords) Métodos pedagógicos activos y globalizadores. Conceptualización y propuestas de aplicación, (85-102). Barcelona. Graó.

Martín-Moya, R. \& Ruiz-Montero, P. J. (2017). «DiverHealth»: motivación en la evaluación de Educación Física. Revista Iberoamericana de Educación, 73(1) 109-120.

Martínez-Franco, C.M. (2017). Gamificación en Educación Física: proyecto Súper Mario Bros. Publicaciones didácticas, 89, 148152.

Melchor, E. (2012). Gamificación y E-Learning: un ejemplo con el juego del pasapalabra. Bruselas: EFQUEL.

Monguillot, M. H., Arévalo, C. G., Mon, C. Z., Batet, L. A. \& Catasús, M. G. (2015). Play the Game: gamificación y hábitos saludables en educación física. Apunts: Educación Física y Deportes, 119, 71-79.

Navarro, D., Martínez, R. \& Pérez-López, I (2017). El enigma de las e EFES: Fortaleza, fidelidad y felicidad. Revista Española de Educación Física y Deportes, 419, 73-85.

Pérez-López, I. (2009). El guardián de la salud: Un juego de rol para promover hábitos nsaludables de vida y actividad física desde la Educación Física Apunts: Educación Física y Deportes, 98, 1522.

Pérez-López, I.J. \& Rivera García, E. (2017). Formar docentes, formar personas: análisis de los aprendizajes logrados por estudiantes universitarios desde una experiencia de gamificación. Signo y Pensamiento 70, 9-114

Pérez-López, I.J.; Rivera García, E. \& Delgado, M. (2017). Mejora de hábitos de vida saludables en alumnos universitarios mediante una propuesta de gamificación. Nutrición Hospitalaria, 34(4):942-951

Pérez-López, I.J., Rivera García, E. y Trigueros Cervantes, C. (2017). «La profecía de los elegidos»: un ejemplo de gamificación aplicado a la docencia universitaria. Revista Internacional de Medicina y Ciencias de la Actividad Física y el Deporte,17(66), 243-260.

Pisabarro, A.M., \& Vivaracho, C.E. (2018). Gamificación en el aula: gincana de programación. ReVisión, 11(1), 85-93

Quintero, L., Jimnénez, F. y Area, M. (2018). Más allá del libro de texto. La gamificación mediada con TIC como alternativa de innovación en Educación Física. Retos, 34, 343-348.

Ripoll, O. (2017). Un joc d'aprenentatges. Els meus onze principis per crear jocs que serveixen per aprendre. Material no publicado

Torres, A., Romero-Rodríguez, L., Pérez-Rodríguez, M.A. \& Björk, S. (2018). Modelo Teórico Integrado de Gamificación en Ambientes E-Learning (E-MIGA). Revista Complutense de Educación, 29(1), 129-145

VV.AA. (2017). Enciclopedia Super Mario Bros $30^{a}$ aniversario. Madrid. Planeta de Agostini.

Werbach, K. \& Hunter, D. (2012). For the Win: How game thinking can revolutionize your business. Philadelphia. Wharton Digital Press. 